\title{
Extension of the Hapke bidirectional reflectance model to retrieve soil water content
}

\author{
G.-J. Yang ${ }^{1,2}$, C.-J. Zhao ${ }^{1}$, W.-J. Huang ${ }^{1}$, and J.-H. Wang ${ }^{1}$ \\ ${ }^{1}$ Beijing Research Center for Information Technology in Agriculture, Beijing, China \\ ${ }^{2}$ State Key Laboratory of Remote Sensing Science, Jointly Sponsored by the Institute of Remote Sensing Applications of \\ Chinese Academy of Sciences and Beijing Normal University, Beijing, China
}

Received: 2 March 2011 - Published in Hydrol. Earth Syst. Sci. Discuss.: 15 April 2011

Revised: 5 July 2011 - Accepted: 14 July 2011 - Published: 19 July 2011

\begin{abstract}
Soil moisture links the hydrologic cycle and the energy budget of land surfaces by regulating latent heat fluxes. An accurate assessment of the spatial and temporal variation of soil moisture is important to the study of surface biogeophysical processes. Although remote sensing has proven to be one of the most powerful tools for obtaining land surface parameters, no effective methodology yet exists for in situ soil moisture measurement based on a Bidirectional Reflectance Distribution Function (BRDF) model, such as the Hapke model. To retrieve and analyze soil moisture, this study applied the soil water parametric (SWAP)-Hapke model, which introduced the equivalent water thickness of soil, to ground multi-angular and hyperspectral observations coupled with, Powell-Ant Colony Algorithm methods. The inverted soil moisture data resulting from our method coincided with in situ measurements $\left(R^{2}=0.867\right.$, RMSE $\left.=0.813\right)$ based on three selected bands $(672 \mathrm{~nm}, 866 \mathrm{~nm}, 2209 \mathrm{~nm})$. It proved that the extended Hapke model can be used to estimate soil moisture with high accuracy based on the field multi-angle and multispectral remote sensing data.
\end{abstract}

\section{Introduction}

The quantification of land surface soil moisture pertains to a large range of applications at various spatial and temporal scales, from climate and weather predictions to agriculture and water management (Liang, 2004). Despite being one of the major hydrological variables, accurate soil moisture data remains largely unavailable, e.g. sparse in-situ measurement networks. The remote sensing of microwave can provide multi-configuration (multi-temporal, multifrequency, multi-

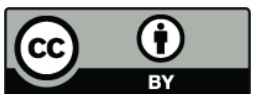

Correspondence to: G.-J. Yang (guijun.yang@163.com) angular, and multi-polarization) radar data that make it possible to address soil moisture estimation and monitoring issues in more effective ways (Baghdadi et al., 2009). Methods use either backscattering model-based retrieval algorithms or polarimetric information derived from target decomposition techniques (Joseph et al., 2009). However, for microwave sensors, which are anyway constrained by very low spatial resolutions and low temporal resolutions. Hence the proposal to use BRDF models on optical data to retrieve soil water content is interesting in this study.

Soil moisture as the key factor influences absorption, reflection and emission characteristics of landsurface, which can directly cause variations in soil energy and water budget (Verhoef and Hapke, 2007). The literatures describe the relationship between soil moisture and the soil reflectance including two main methods as follows: (a) only parameters related to soil moisture are retained, while other factors influencing soil spectra are either fixed or neglected. The model is obtained by regression analysis on the observation data of surface soil moisture and the soil characteristic spectrum. For example, Bach and Mauser (1994) proposed the soil spectral response models of the three water-absorption spectrum bands $(1430 \sim 1450 \mathrm{~nm}, 1620 \sim 1650 \mathrm{~nm}$ and $1920 \sim$ $1940 \mathrm{~nm}$ ) under different soil moisture conditions, focusing only on the effects of scattering and surface water absorption of soil particles. Liu et al. (2004) adopted the methods of relative reflectance, first-order differential and difference in the prediction and modeling of soil surface moisture. (b) Principal component analysis is also used to model soil moisture parameters and reflectance spectra. Price (1990) conducted a large number of measurements and analyses on the reflection spectrum of soil samples, and 10 base vectors were obtained to describe the entire reflection spectrum database approximately. Other studies extracted four base functions from the base vectors to describe the entire reflection spectrum of the soil using principal component analysis (Cierniewski, 1987; Bach and Mauser, 1994; Fabiano and David, 2010). Finally,

Published by Copernicus Publications on behalf of the European Geosciences Union. 
Walthall et al. (1985) proposed an empirical model for the calculation of soil reflectance that only considered solar incidence and observation direction parameters of the sensor. However, the empirical model was difficult to use directly for surface or remote sensing inversions due to its limited applicability under laboratory conditions.

Bidirectional Reflectance Distribution Function (BRDF) modeling does not consider soil moisture (Pinty et al., 1989), and this restricts the model to use soil moisture inversions from remote sensing data. In a geometrical optics model, the proportion of shadow area due to rough soil surface under direct sunlight in the visible field changes when the sensor observes from different directions ( $\mathrm{Li}$ and Strahler, 1992), and the root mean square and correlation coefficient of the soil roughness height are the only two parameters typically considered (Norman et al., 1985; Pinty and Verstraete, 1991; Jacquemoud et al., 1992; Verhoef and Hapke, 2007). For example, Cierniewski et al. (1987) simulated soil as a series of regular and opaque spheres with equivalent particles on a smooth surface and then developed the series model based on geometric optical models. Aimed at solving complex problems, such as the shape, size, orientation and heterogeneous scattering of soil particles, volume-scattering effect models, among which the Hapke model (Hapke, 1981) has achieved recognition, are based on the radiative transfer theory. In the Hapke model, the surface soil radiance received by the sensor consists of two parts: single scattering and multiple scattering. The significant hotspot effect of soil BRDF requires the addition of a correction term. Given that the assumption of multiple scattering satisfies isotropy, the method of twostream approximation obtains a numerical solution. Later, scientists further developed and improved the Hapke model, using the new models to study physical properties of the soil. They claimed the new models, including the SOILSPECT model developed by Jaquemoud et al. (1992), were more accurate than the Hapke model. Regarding model application, Chappell et al. (2006) used the Hapke model for the directional modeling of soil radiation with heavy rain and wind erosion; Wu et al. (2009) combined the Hapke model with MISR data to conduct a comparative analysis on the model parameters of different sandy land types in China's western desert areas, proving the model's good accuracy. In addition, Cooper et al. (1985) used the Monte Carlo Computing Strategy to simulate soil surface radiation properties and found that a great number of calculations and probability statistics were necessary to obtain a stable pattern and that great uncertainties existed in the model parameter solution.

The variation of scattering and absorption on the particle surface caused by the soil moisture changes the soil reflectance of the dual-hemisphere, which has a quantitative conversion with the single scattering albedo of the SOILSPECT model. In this study, the parameters of soil water content were introduced into the soil BRDF model to establish the extended soil BRDF model. Bare farmlands were selected to conduct multi-angle spectral measurements and

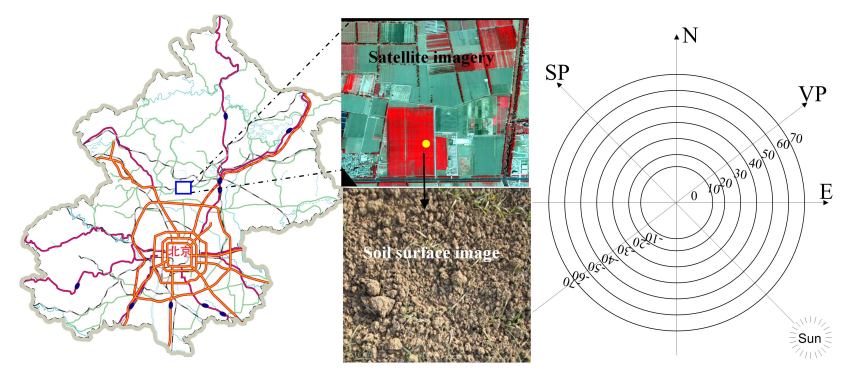

Fig. 1. The location of the study area, soil surface image and observation orientations; the satellite imagery is a Landsat 5 TM image (R:5, G:4, B:3) on 18 May 2009.

soil water content measurements. All the model parameters were inverted by the combination of the Ant Colony Algorithm and Powell's Method (Matlah, 2000; Dorigo et al., 1996, 2006), and lastly, the model estimation results of the characteristic band were analyzed and verified.

\section{Materials and methods}

\subsection{The experimental area and data acquisition}

The National Precision Agriculture Research and Demonstration Base served as the experimental area in the northern part of Xiaotangshan Town, Changping District, Beijing, featuring a ground slope of $1 / 1500$ and the base boundaries within $40^{\circ} 10^{\prime} 31^{\prime \prime}-40^{\circ} 11^{\prime} 18^{\prime \prime} \mathrm{N}, 116^{\circ} 26^{\prime} 10^{\prime \prime}-116^{\circ} 27^{\prime} 05^{\prime \prime} \mathrm{E}$, covering an area of $167 \mathrm{ha}$. The annual average ground temperature is approximately $14.5^{\circ} \mathrm{C}$; the maximum frozen soil depth is $0.8 \mathrm{~m}$; yellow clay is distributed in the soil at a depth of $0 \sim 4 \mathrm{~m}$ with a bulk density of $1.45 \mathrm{~g} \mathrm{~cm}^{-3}$. The ASD Fieldspec FR2500 spectrometer $(350-2500 \mathrm{~nm})$ and a simple multi-angle observation scaffold were adopted for the multi-angle spectral measurements (Fig. 1). The field angle of the spectrometer probe was $25^{\circ}$, and the probe height was $185 \mathrm{~cm}$ from the surface for observing vertically downwards with a corresponding ground range of $30 \mathrm{~cm} \times 30 \mathrm{~cm}$. The reference plate was the whiteboard with a reflectance of $30 \%$. Two sample sites were set (Sites 1 and 2) for four sets of multi-angle spectral observations in which two orientations of the solar principal plane (SP) and the vertical solar principal plane (VP) were measured with a view zenith angle ranging between $-70^{\circ}$ and $70^{\circ}$ for each orientation and a step interval of $10^{\circ}$ (Fig. 1). Total measured multi-angle spectral data were resampled into $10 \mathrm{~nm}$ wavelength intervals to be more consistent with hyperspectral sensors. The positive view zenith angle indicated the backward reflection of the observation surface, and the negative view zenith angle corresponded to the forward reflection of the observation surface. The observation time was 09:00 12:00 a.m. UTC+8, on 27 October 2009, when the weather exhibited stability 
without clouds, good atmospheric visibility and a wind speed of $5 \mathrm{~m} \mathrm{~s}^{-1}$.

The soil type was the same throughout the experimental area. Time domain reflectometry (TDR300) was used for soil water content measurement, and the values of soil volumetric water content (VWC) were recorded using the TDR measurements [unit: $100 \times\left(\mathrm{m}^{3} \mathrm{~m}^{-3}\right)$ ] with a $20-\mathrm{cm}$ probe. In each set of multi-angle spectral observation sites, the VMC measurements were conducted 8 times continuously, and the mean of the values was used for corresponding soil water content.

\subsection{Soil BRDF model and its extension}

The Hapke model is a widely-used soil bi-directional reflectance model (Verhoef and Hapke, 2007; Wu et al., 2009). The model considers that the horizontal surface $(z=0)$ contains irregular soil particles with surfaces facing random directions and that the particle size is larger than the wavelength. The soil receives the incident solar radiation with a direction of $\left(\theta_{\mathrm{s}}, \phi\right)$ and an intensity, $J$, that a sensor observes with a direction of $\left(\theta_{0}, 0\right)$. The scattering angle, $g$, refers to the angle between incident light and emergent light. Among these variables, $\theta_{\mathrm{s}}, \theta_{\mathrm{o}}$ are the solar zenith angle and the view zenith angle, respectively, and $\phi$ is the relative azimuth between the sun and the observation direction. The radiation $I$ observed by the sensor is the sum of the single scattering part $I_{\mathrm{s}}$ and the multiple scattering $I_{\mathrm{m}}$ :

$I=I_{\mathrm{s}}+I_{\mathrm{m}}$

where, $I_{\mathrm{S}}=\frac{J \omega}{4 \pi} \frac{\cos \theta_{\mathrm{s}}}{\cos \theta_{\mathrm{s}}+\cos \theta_{\mathrm{o}}} P(g)$

$I_{\mathrm{m}}=\frac{J \omega}{4 \pi} \frac{\cos \theta_{\mathrm{s}}}{\cos \theta_{\mathrm{s}}+\cos \theta_{\mathrm{o}}}\left[H\left(\cos \theta_{\mathrm{s}}\right) H\left(\cos \theta_{\mathrm{o}}\right)-1\right]$

where $H(x)=\frac{1+2 x}{1+2 x \sqrt{1-\omega}}, \omega$ is the single scattering albedo of soil, and $P(g)$ is the scattering phase function. For a semifinite medium, Cierniewski (1987) shows that the sensitivity of the single-scattering part $I_{\mathrm{S}}$ on the scattering phase function of the particles is greater than that of the multiple scattering part $I_{\mathrm{m}}$ on the scattering phase function. Therefore, the actual values are used in the scattering phase function for the calculation of single scattering, but in the calculation of multiple scattering, it is assumed that all the scatterings are uniform in each direction, or $P(g)=1$.

The SOILSPECT model is an improvement of the Hapke model. According to the Legendre polynomial, a scattering phase function $P\left(g, g^{\prime}\right)$ is proposed to interpret both backward scattering and forward scattering from a smooth soil surface. In this function, $g^{\prime}$ indicates the angle between the direction of spectral reflection and the direction of incident light:

$$
\begin{aligned}
& P\left(g, g^{\prime}\right)=1+b \cos (g)+\frac{c\left(3 \cos ^{2}(g)-1\right)}{2}+ \\
& b^{\prime} \cos \left(g^{\prime}\right)+\frac{c^{\prime}\left(3 \cos ^{2}\left(g^{\prime}\right)-1\right)}{2}
\end{aligned}
$$

where, $\cos (g)=\cos \left(\theta_{\mathrm{S}}\right) \cos \left(\theta_{\mathrm{o}}\right)+\sin \left(\theta_{\mathrm{S}}\right) \sin \left(\theta_{\mathrm{O}}\right) \cos (\phi)$

$\cos \left(g^{\prime}\right)=\cos \left(\theta_{\mathrm{S}}\right) \cos \left(\theta_{\mathrm{O}}\right)-\sin \left(\theta_{\mathrm{S}}\right) \sin \left(\theta_{\mathrm{O}}\right) \cos (\phi)$

In the equation, $b, c, b^{\prime}$ and $c^{\prime}$ are coefficients of the scattering phase function.

The soil is a discontinuous system composed of blocks separated by pores in the Hapke model, requiring the introduction of the backward scattering function $B(g)$. This function contains three parameters: the scattering angle $g$, the half-width parameters of the hot spot effect (roughness) $h$ and the parameter $B_{0}$ describing the intensity size of the hot spot effect. Thus, the soil bidirectional reflectance model (SOILSPECT) can be expressed as follows:

$$
\begin{aligned}
& I=\frac{J \omega}{4 \pi} \frac{\cos \theta_{\mathrm{s}}}{\cos \theta_{\mathrm{s}}+\cos \theta_{\mathrm{o}}} \\
& \left\{P\left(g, g^{\prime}\right)[1+B(g)]+H\left(\cos \theta_{\mathrm{s}}\right) H\left(\cos \theta_{\mathrm{o}}\right)-1\right\} \\
& \text { where, } B(g)=\frac{B_{\mathrm{o}}}{1+\tan (g / 2) / h} .
\end{aligned}
$$

For the given scattering angle $\left(g, g^{\prime}\right)$, this model shows that the enlargement of roughness $h$ will lead to the increase of backscattering. Based on the Eq. (3), the soil bidirectional reflectance $r\left(\theta_{\mathrm{s}}, \theta_{\mathrm{o}}, \phi\right)$ can be obtained as:

$$
\begin{aligned}
& r\left(\theta_{\mathrm{s}}, \theta_{\mathrm{o}}, \phi\right)=\frac{\omega}{4} \frac{1}{\cos \theta_{\mathrm{s}}+\cos \theta_{\mathrm{o}}} \\
& \left\{P\left(g, g^{\prime}\right)[1+B(g)]+H\left(\cos \theta_{\mathrm{s}}\right) H\left(\cos \theta_{\mathrm{o}}\right)-1\right\}
\end{aligned}
$$

To introduce the parameters of soil water content into the SOILSPECT model, with consideration of the impact of soil moisture, it is assumed that the equivalent water thickness $\xi$ corresponds to the variation from $r_{0}$ to $r_{\mathrm{w}}$ of the hemisphere reflection of dry soil due to soil moisture. In other words, the extinction contribution corresponding to the saturated liquid water with the thickness of $\xi$ is equivalent to the total soil extinction contribution of a given soil water content, including multiple scattering and absorption. The Beer-Lambert law is used to calculate the dual-hemisphere reflectance through the extinction effect of soil moisture, as follows:

$r_{\mathrm{W}}=r_{0} * \exp (-\alpha \cdot \xi)$

In the equation, $\alpha$ is the absorption coefficient $\left(\mathrm{cm}^{-1}\right), \xi$ is the equivalent water thickness of soil moisture $(\mathrm{cm}), r_{0}$ is the sampling dry soil reflectance, and $r_{\mathrm{w}}$ is the doublehemisphere reflectance of wet soil. Among them, the water absorption coefficient $\alpha$ is the reference value within the wavelength range of $0.4-2.5 \mu \mathrm{m}$ measured by Segelstein's laboratory (Segelstein, 1981) (Fig. 2). $r_{0}$ is directly obtained from the soil sample dried with a spectrometer and measured in the laboratory, and the dried sample is maintained consistent with the original surface condition. According to the definition of $r_{\mathrm{w}}$, it is only influenced by the physical properties of the soil and exhibits no relationship to the observation direction in theory. If the multi-angle spectral data are used for the calculation of equivalent water thickness according to 


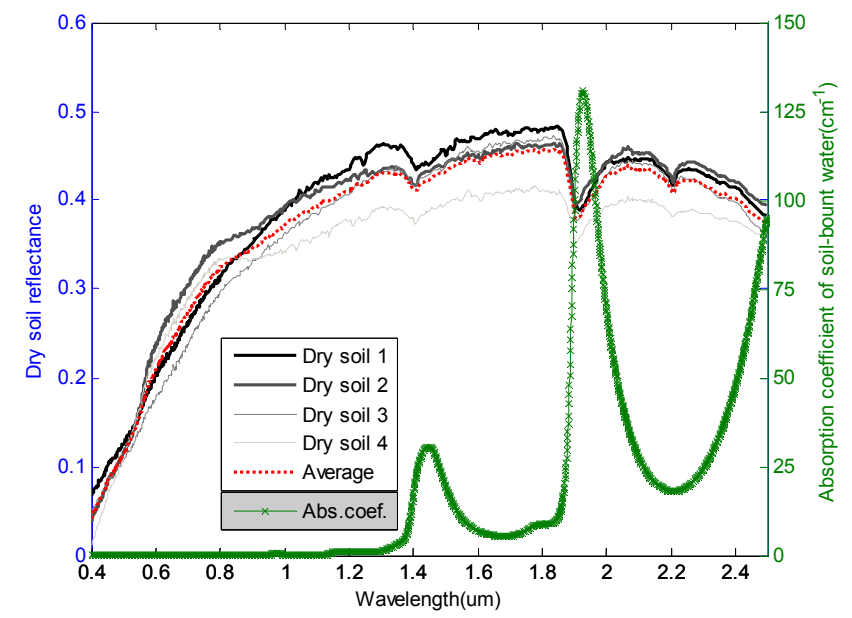

Fig. 2. The coefficient of water absorption and reflectance of dry soil.

Eq. (5), $\xi$ will vary. However, the $\xi$ value is actually unique for the same soil sample, and a relationship should be established between $r_{\mathrm{w}}$ and the parameters of soil optical properties in the SOILSPECT model. Finally, the real equivalent water thickness is estimated using the same set of multi-angle spectral data.

Bach and Mauser (1994) proposed an equation describing the relationship between bi-hemisphere reflectance and single scattering albedo:

$\omega=\left[1-\left(\frac{1-r_{\mathrm{w}}}{1+r_{\mathrm{w}}}\right)^{2}\right] /\left[1+\frac{b}{4}\left(\frac{1-r_{\mathrm{w}}}{1+r_{\mathrm{w}}}\right)^{2}\right]$

Because Eqs. (4), (5) and (6) were established simultaneously, the extension of the original soil BRDF model was complete. The parameters of soil water content were introduced into the model renamed the SWAP-Hapke model, convenient for the estimation of all model parameters including soil water content directly using multi-angle observation data. With these modifications, the estimation accuracy of soil moisture can be improved with the utilization of multiangle spectral data.

\subsection{Model parameter inversion and evaluation methods}

Because the extended soil BRDF model is nonlinear, the adoption of Powell's Method effectively avoids the uncertainties of model derivation, but Powell's Method depends on the initial points. Powell's method, strictly Powell's conjugate gradient descent method, is an algorithm proposed by Michael J. D. Powell for finding a local minimum of a function. The function need not be differentiable, and no derivatives are taken (Matlab, 2000). The number of model parameters requiring a solution prompted the selection of the Ant Colony Algorithm (ACA) to obtain a better solution for the optimal initial value of Powell's Method and through it obtaining the local optimal solution of model parameters (Matlab, 2000). The ACA is a robust simulated evolutionary algorithm based on the collective behaviors of real ant colonies in nature and featuring a positive feedback mechanism (Dorigo et al., 1996). In general, the ACA produces relatively satisfactory results, but it may produce a suboptimal solution adjacent to global optimal solution rather than the global optimal solution itself. Powell's Method demonstrates improvement over the coordinate alternation method, or directional acceleration method, that requires no derivative calculations of the objective function. The iterative search of the Brent algorithm was adopted for each dimension with the quadratic convergence, thereby increasing the search speed with a strong local optimization capacity and the accuracy compared to other optimization algorithms.

This study combines these two optimization algorithms. The optimal solution of the parameters is a search for the optimal solution of the parameters to be solved $\left(\omega, B_{0}, h, b, c, b^{\prime}, c^{\prime}, \xi\right)$ with the SWAP-Hapke soil BRDF model as a constraint equation, that is, $r=$ $f_{\text {SWAP-Hapke_BRDF }}\left(\omega, B_{0}, h, b, c, b^{\prime}, c^{\prime}, \xi\right)$ to achieve the minimized index function $P$.

$P=\sum_{k=1}^{n}\left[r_{k}-r\left(\theta_{\mathrm{s}}, \theta_{\mathrm{o}}, \phi\right)\right]^{2}$

where $n$ is the number of combinations of multi-angle spectral observation angles, $r_{k}$ is the multi-angle spectral observation value with the view azimuth of $\left(\theta_{\mathrm{s}}, \theta_{\mathrm{o}}, \phi\right)$, and $r$ is the simulated value of the SWAP-Hapke model with the given parameters of preparative optimization. The boundary range of model parameters and the main algorithm parameters in the optimization solution are shown in Table 1, and default values are set for other parameters:

Based on the inversion method from previous studies (Pinty et al., 1991; Jacquemoud et al., 1992; and Chappell et al., 2006), five bands, including $G(558 \mathrm{~nm}), \mathrm{R}$ $(672 \mathrm{~nm})$, NIR $(866 \mathrm{~nm})$, SWIR1 $(1570 \mathrm{~nm})$ and SWIR2 $(2209 \mathrm{~nm})$, were selected for the estimation of model parameters $\omega, B_{0}, h, b, c, b^{\prime}, c^{\prime}, \xi$. To verify the reliability in estimating the model parameters, the multi-angle spectral observation was conducted 4 times in each sample area, and the data parameter estimation was carried out in accordance with two programs: adopting the multi-angle spectral data of the single band measured from the surface in the estimation of model parameters to obtain the estimation results of the parameters at 5 wavelengths and adopting the multi-angle spectral data of the three characteristic bands (R-NIR-SWIR2) in one set of measurement data (R-NIR-SWIR2) in the estimation of model parameters to obtain those suitable for multiangle and multi-spectral data. The regression analysis and accuracy comparison were conducted on the obtained equivalent water thickness at different wavelengths and the measured soil water content.

The following indicators were adopted to assess accuracy (Li and Wang, 1995; Liang, 2004; Fuwei et al., 2009): 
Table 1. The setting of parameters for the SWAP-Hapke model and the initialization of the ACA and Powell algorithm.

\begin{tabular}{|c|c|c|c|}
\hline \multicolumn{2}{|l|}{ SWAP-Hapke model } & \multicolumn{2}{|l|}{ ACA and Powell algorithm } \\
\hline Parameters & Value range & Parameters & Value range \\
\hline Single scattering albedo $(\omega)$ & $0-1$ & The relative importance of the trail for ACA $(\alpha)$ & $0-5$ \\
\hline Soil surface roughness $(h)$ & $0-1.5$ & The relative importance of the visibility for ACA $(\beta)$ & $0-5$ \\
\hline Hot spot effect $\left(B_{0}\right)$ & $0-1$ & The trail persistence for $\operatorname{ACA}(\rho)$ & $0.1-0.99$ \\
\hline The coefficient of the scattering phase function $(b)$ & $-2-2$ & the quantity of trail laid by ants for $\operatorname{ACA}(Q)$ & $10-100000$ \\
\hline The coefficient of the scattering phase function $(c)$ & $-1-1$ & The number of ants for ACA $(m)$ & 40000 \\
\hline The coefficient of the scattering phase function $\left(b^{\prime}\right)$ & $-2-2$ & The number of tails for ACA $(l)$ & 1000 \\
\hline The coefficient of the scattering phase function $\left(c^{\prime}\right)$ & $-1-1$ & The number of maximal iteration for Powell $\left(N_{c \max }\right)$ & 10000 \\
\hline
\end{tabular}

1. Root mean square error (RMSE), representing the overall distribution of errors between the model estimates and actually observed values:

$$
\mathrm{RMSE}=\sqrt{\frac{\sum_{i=1}^{n}\left(e_{i}-o_{i}\right)^{2}}{n_{f}}}
$$

In the equation, $n$ is the total number of observations, and $n_{f}$ is the number of redundant observations, i.e., the difference between the total number of observations $n$ and the number of model parameters to be solved; $e_{i}$ and $o_{i}$ are the model estimate and the observed value, respectively.

2. The Nash-Sutcliffe efficiency coefficient $(E)$ expresses the consistency of overall sample errors between the estimates and the observed values represented by the commonly used coefficient of determination $R^{2}$ and indicates the distribution consistency of internal sample errors. Within a range of $-\infty \sim 1, E=1.0$ represents the model estimates completely consistent with the observed values; $E=0.0$ represents the accuracy of model estimates reaching the average level of the observed values; and $E<0.0$ indicates a relatively great error in the model estimates.

$$
E=1.0-\frac{\sum_{i=1}^{n}\left|e_{i}-o_{i}\right|}{\sum_{i=1}^{n}\left|e_{i}-\bar{o}_{i}\right|}
$$

\section{Results and discussions}

\subsection{Inversion results of the model parameters}

Model parameters were retrieved from simulated data (Mode 1). Due to the nonlinearity of the SWAP-Hapke model, providing parameter values in it enables the calculation of unique results of the soil BRDF distribution, but its unique solution may not be available at a given set of soil BRDF observation data. Therefore, to ensure an accurate solution of model parameters, a set of model parameter values $\left(\omega=0.25, B_{0}=0.4, h=0.05, b=1.0, b^{\prime}=0.25, c^{\prime}=-0.2\right.$ and $c=-0.5)$ were selected with references from Pinty et al. (1989). With 40 sets of random errors of $0 \sim 5 \%$ added to the simulated model data, the model parameters were recalculated using the ACA and Powell's Algorithm, and the distributions of the RMSE parameters are shown in Fig. 3.

Figure 3 indicates that the values of $\omega$ and $B_{0}$ were more consistent with their mean of 0.40 , although $h$ showed a relatively great fluctuation, especially when the RMSE value was greater than 0.01 . In this study, $\omega$ and $b$ were the main parameters influencing the accurate estimation of soil water content; therefore, when the RMSE value was within 0.02 , the estimation errors of the extended model parameters met the estimation requirements of soil moisture parameters. Second, the error analysis results in Fig. 3 illustrate the smaller stabilities of $b$ and $b^{\prime}$ compared to those of $c$ and $c^{\prime}$ with different RMSE values. The impact of the errors was especially elevated when the RMSE was greater than 0.015. Generally, when the RMSE value was smaller than 0.02 , the relationship among the parameters followed the order: $b>b^{\prime}>c^{\prime}$ $>c$. In addition, the size of difference between $b$ and $b^{\prime}$ is twice greater than that between $c$ and $c^{\prime}$. Finally, Fig. 3 indicates that the errors of $\omega$ and $b$ significantly influenced the error of $\xi$.

Model parameters were retrieved from in-situ measurements (Mode 2). The SWAP-Hapke model parameters were calculated by the methods described in Sect. 2.3 from three sets of multi-angle surface observation data in Site 1 and Site 2 (Table 2). The estimation results of the model parameters at different bands showed an order of $\mathrm{E}(\mathrm{NIR})>\mathrm{E}(\mathrm{SWIR} 2)>\mathrm{E}(\mathrm{R})>\mathrm{E}(\mathrm{SWIR} 1)>\mathrm{E}(\mathrm{G})$ from the accuracy index $\mathrm{E}$ of overall parameter estimation, where 8 sets of means for different bands of E(NIR), E(SWIR2) and $E(R)$ were greater than 0.8 . This indicates that the three bands were more suitable for estimating model parameters. 

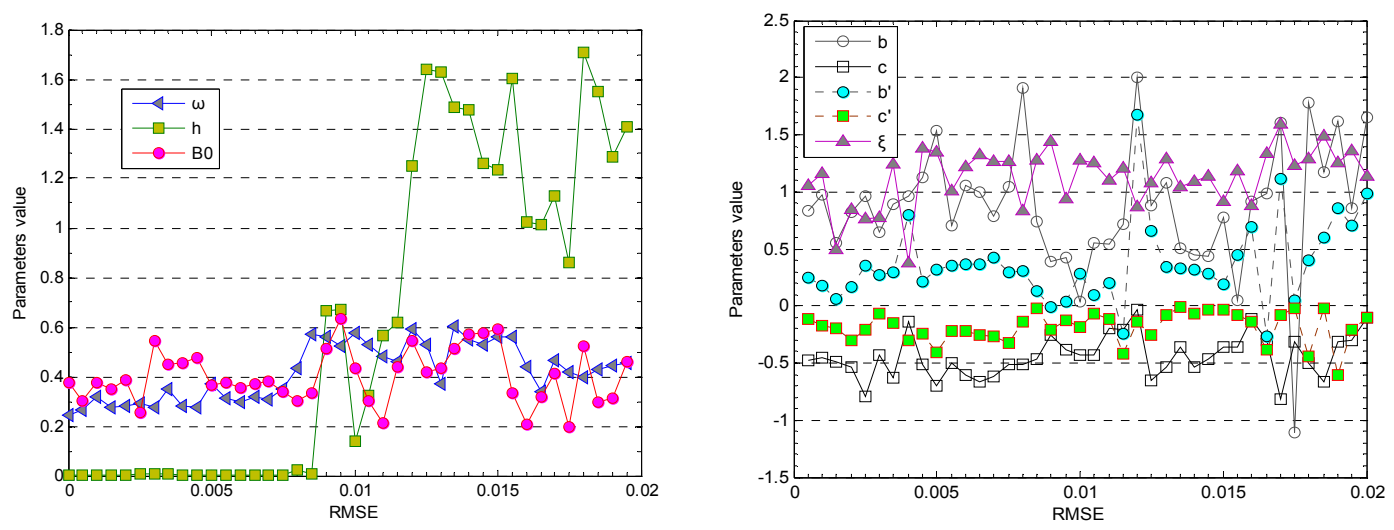

Fig. 3. The results of the SWAP-Hapke parameters and the distribution of RMSE based on simulated data.

The mean of $\mathrm{E}(\mathrm{G})$ was approximately 0.6 , suggesting a relatively great error of parameter estimates, although the mean of $\mathrm{E}(\mathrm{SWIR} 1)$ was higher than that of $\mathrm{E}(\mathrm{G})$. The following three aspects categorize the main differences in the accuracies of each estimation in different bands: (1) some differences in bands were influenced by the absorption and scattering of atmospheric $\mathrm{H}_{2} \mathrm{O}$ or $\mathrm{O}_{2}$, with better results in the NIR and R-band than in the other three bands; (2) differences in the sensitivity of soil moisture absorption occurred in different bands; and (3) different levels of noise appeared in the surface spectrometer at different wavelengths, and the noise equivalent radiance (NEDL) increased with longer wavelengths. The reasonable differences appearing in $\omega$, which was solved with 8 sets of multi-angle spectral observations in Site 1 and Site 2, proved that the spatial variability of the soil moisture distribution was the main factor influencing the results. This conclusion was further supported by the subsequent analysis of soil equivalent water thickness $\xi$ and the actual measurements of soil water content. The estimation results of the model parameters at different bands also indicated that the four parameters of $b, c, b^{\prime}$ and $b^{\prime}$ influenced the forward and backward scattering of the soil. When $b$ and $c$ were both greater than $b^{\prime}$ and $c^{\prime}$, the soil mainly exhibited backscattering, but the soil may have shown forward scattering, when $b$ and $c$ were both less than $b^{\prime}$ and $c^{\prime}$. When their values were nearly the same, the soil demonstrated the characteristics of a combination of forward and backward scattering. When $b>b^{\prime}>\left|c^{\prime}\right| \approx|c|$, the soil BRDF displayed the characteristics of backward scattering. Among the four coefficients of scattering phase functions, the variation range of $b$ contributed the most. Because of the close relationship among $\xi, \omega$ and $b$, the variations in the estimated $\xi$ in all bands were in a range between $\omega$ and $b$, and neither the 8 sets of equivalent water thickness $\xi$ estimated by the model nor the actual TDR measurements represented the soil water content of Site 1 and Site 2.

\subsection{Validation of the estimated model parameters}

Based on the single-band estimation, the SWAP-Hapke model parameters were obtained by adopting the combined solution of the three optimal bands (R, NIR and SWIR2) from the first three sets of multi-angle data in Site 1 and Site 2. Aimed at the further assessment on estimation accuracy of combined multi-band model parameters, a set of multi-angle spectral data of the vertical solar principal plane and the solar principal plane were simulated and compared with the fourth set of multi-angle observation values.

The results (Fig. 4) show that the model-simulated values were consistent with the observed values, with the exception of the error in the SWIR1 band in the vertical solar principal plane. The BRDF from the other bands in this principal plane were maintained as the "bowl edge" shape, consistent with the model simulation. Figure 4 also indicates that the consistency between the model output values and the measured values in the solar principal plane improved over that in the vertical solar principal plane. The BRDF increased as the observation angle gradually approached the zenith of the solar incidence angle from the nadir. When the zenith of the solar angle neared $70^{\circ}$ in the actual observations and the maximum observation angle of the multi-angle observation scaffold reached $70^{\circ}$, the hot spot effect appeared without an obvious hot spot width. In addition, the following conditions might influence the estimation accuracy of the model parameters: (1) in a wide-angle observation, the surface range of a multi-angle observation is significantly greater than the observation range from the nadir, and (2) a slight fluctuation on the surface may impact the results, despite the roughness of the farmland terrain owing to common irrigation practices.

The estimated equivalent water thickness $\xi$ was quite different from the soil water content and TDR measurements in the agronomy. The soil water content at a depth up to $50 \mathrm{~cm}$ was required for the winter wheat because its roots were primarily distributed in this depth range. Given that the this study was carried out on the bare farmland soil, where 
Table 2. The estimated results for Site 1 and Site 2 based on single band.

\begin{tabular}{|c|c|c|c|c|c|c|c|c|c|c|}
\hline & $\omega$ & $h$ & $B_{0}$ & $b$ & $c$ & $b^{\prime}$ & $c^{\prime}$ & $\xi$ & RMSE & E \\
\hline \multicolumn{11}{|c|}{ G band } \\
\hline \multirow[t]{4}{*}{ Site 1} & 0.244 & 0.004 & 0.377 & 0.837 & -0.477 & 0.253 & -0.116 & 1.083 & 0.015 & 0.638 \\
\hline & 0.268 & 0.005 & 0.304 & 0.972 & -0.452 & 0.180 & -0.175 & 1.287 & 0.010 & 0.747 \\
\hline & 0.348 & 0.003 & 0.375 & 0.558 & -0.489 & 0.057 & -0.198 & 1.042 & 0.011 & 0.732 \\
\hline & 0.276 & 0.003 & 0.350 & 0.825 & -0.532 & 0.163 & -0.303 & 1.09 & 0.016 & 0.661 \\
\hline \multirow[t]{4}{*}{ Site 2} & 0.283 & 0.001 & 0.389 & 0.959 & -0.796 & 0.358 & -0.213 & 1.134 & 0.016 & 0.671 \\
\hline & 0.294 & 0.009 & 0.254 & 0.648 & -0.424 & 0.268 & -0.062 & 0.918 & 0.012 & 0.759 \\
\hline & 0.217 & 0.007 & 0.547 & 0.886 & -0.625 & 0.293 & -0.151 & 1.179 & 0.009 & 0.800 \\
\hline & 0.351 & 0.008 & 0.448 & 0.963 & -0.135 & 0.798 & -0.303 & 0.881 & 0.020 & 0.581 \\
\hline \multicolumn{11}{|c|}{$\mathrm{R}$ band } \\
\hline \multirow[t]{4}{*}{ Site 1} & 0.284 & 0.003 & 0.455 & 1.130 & -0.514 & 0.218 & -0.238 & 1.334 & 0.007 & 0.883 \\
\hline & 0.274 & 0.005 & 0.474 & 1.535 & -0.693 & 0.320 & -0.411 & 1.595 & 0.005 & 0.894 \\
\hline & 0.370 & 0.004 & 0.367 & 0.706 & -0.505 & 0.350 & -0.225 & 1.234 & 0.005 & 0.874 \\
\hline & 0.313 & 0.001 & 0.377 & 1.057 & -0.610 & 0.361 & -0.219 & 1.287 & 0.007 & 0.836 \\
\hline \multirow[t]{4}{*}{ Site 2} & 0.299 & 0.002 & 0.357 & 0.992 & -0.668 & 0.365 & -0.255 & 1.487 & 0.013 & 0.642 \\
\hline & 0.318 & 0.004 & 0.370 & 0.784 & -0.615 & 0.419 & -0.264 & 1.259 & 0.009 & 0.771 \\
\hline & 0.306 & 0.002 & 0.379 & 1.045 & -0.515 & 0.298 & -0.329 & 1.354 & 0.009 & 0.765 \\
\hline & 0.352 & 0.003 & 0.338 & 1.912 & -0.512 & 0.309 & -0.133 & 1.142 & 0.017 & 0.597 \\
\hline \multicolumn{11}{|c|}{ NIR band } \\
\hline \multirow[t]{4}{*}{ Site 1} & 0.404 & 0.025 & 0.305 & 0.739 & -0.468 & 0.127 & -0.016 & 1.278 & 0.003 & 0.906 \\
\hline & 0.389 & 0.006 & 0.333 & 0.387 & -0.257 & -0.006 & -0.202 & 1.439 & 0.003 & 0.903 \\
\hline & 0.451 & 0.666 & 0.513 & 0.422 & -0.385 & 0.040 & -0.126 & 0.941 & 0.004 & 0.891 \\
\hline & 0.423 & 0.673 & 0.633 & 0.038 & -0.425 & 0.287 & -0.183 & 1.272 & 0.005 & 0.870 \\
\hline \multirow[t]{4}{*}{ Site 2} & 0.398 & 0.138 & 0.433 & 0.552 & -0.425 & 0.096 & -0.073 & 1.253 & 0.007 & 0.834 \\
\hline & 0.453 & 0.323 & 0.303 & 0.545 & -0.199 & 0.204 & -0.118 & 1.102 & 0.007 & 0.833 \\
\hline & 0.421 & 0.566 & 0.212 & 0.716 & -0.212 & -0.241 & -0.417 & 1.205 & 0.003 & 0.927 \\
\hline & 0.463 & 0.620 & 0.440 & 2.005 & -0.032 & 1.672 & -0.143 & 0.868 & 0.012 & 0.610 \\
\hline \multicolumn{11}{|c|}{ SWIR1 band } \\
\hline \multirow[t]{4}{*}{ Site 1} & 0.541 & 1.251 & 0.544 & 0.883 & -0.654 & 0.659 & -0.250 & 1.052 & 0.016 & 0.683 \\
\hline & 0.53 & 1.642 & 0.419 & 1.082 & -0.540 & 0.341 & -0.083 & 1.156 & 0.015 & 0.730 \\
\hline & 0.571 & 1.627 & 0.433 & 0.511 & -0.364 & 0.328 & -0.009 & 0.499 & 0.011 & 0.780 \\
\hline & 0.562 & 1.485 & 0.514 & 0.453 & -0.531 & 0.319 & -0.065 & 0.843 & 0.010 & 0.792 \\
\hline \multirow[t]{4}{*}{ Site 2} & 0.552 & 1.475 & 0.573 & 0.432 & -0.465 & 0.289 & -0.028 & 0.765 & 0.009 & 0.833 \\
\hline & 0.589 & 1.258 & 0.577 & 0.778 & -0.363 & 0.189 & -0.036 & 0.779 & 0.012 & 0.713 \\
\hline & 0.563 & 1.235 & 0.594 & 0.053 & -0.361 & 0.445 & -0.080 & 1.237 & 0.013 & 0.745 \\
\hline & 0.592 & 1.603 & 0.332 & 0.912 & -0.112 & 0.690 & -0.133 & 0.382 & 0.018 & 0.618 \\
\hline \multicolumn{11}{|c|}{ SWIR2 band } \\
\hline \multirow[t]{4}{*}{ Site1 } & 0.441 & 1.024 & 0.206 & 0.991 & -0.301 & -0.263 & -0.385 & 1.378 & 0.007 & 0.865 \\
\hline & 0.357 & 1.015 & 0.320 & 1.604 & -0.816 & 1.118 & -0.077 & 1.345 & 0.004 & 0.899 \\
\hline & 0.467 & 1.131 & 0.412 & -1.109 & -0.317 & 0.047 & -0.020 & 1.009 & 0.006 & 0.876 \\
\hline & 0.416 & 0.860 & 0.200 & 1.775 & -0.499 & 0.400 & -0.444 & 1.225 & 0.007 & 0.855 \\
\hline \multirow[t]{4}{*}{ Site2 } & 0.398 & 1.708 & 0.526 & 1.177 & -0.669 & 0.594 & -0.019 & 1.322 & 0.010 & 0.756 \\
\hline & 0.429 & 1.548 & 0.300 & 1.618 & -0.312 & 0.862 & -0.611 & 1.265 & 0.011 & 0.780 \\
\hline & 0.443 & 1.287 & 0.312 & 0.859 & -0.300 & 0.701 & -0.210 & 1.268 & 0.010 & 0.791 \\
\hline & 0.455 & 1.406 & 0.461 & 1.655 & -0.107 & 0.990 & -0.106 & 0.836 & 0.013 & 0.710 \\
\hline
\end{tabular}




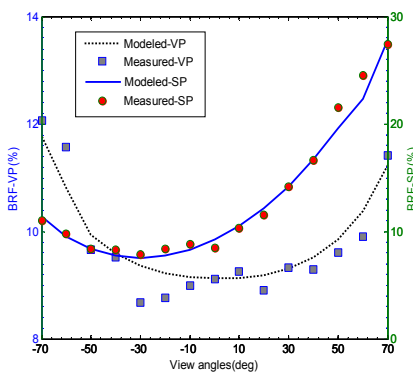

(a) G band

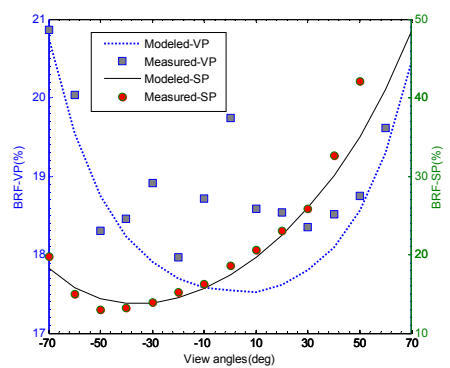

(d) SWIR1 band

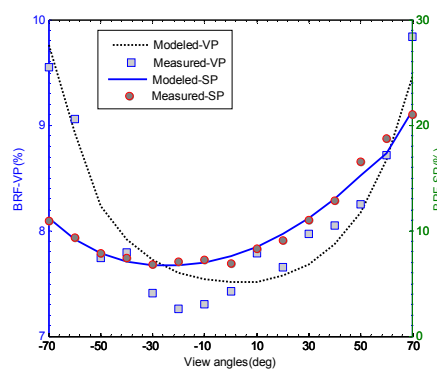

(b) R band

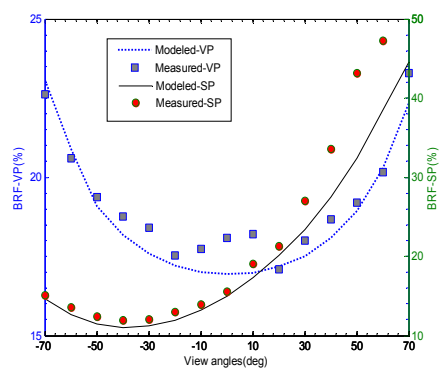

(e) SWIR2 band

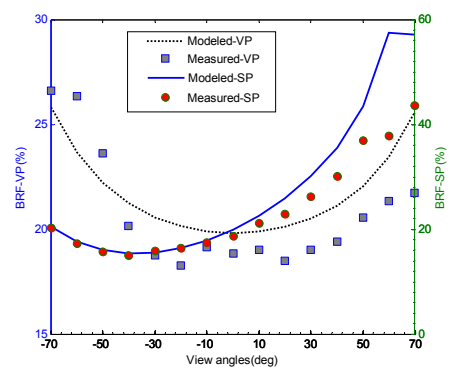

(c) NIR band

Fig. 4. The validation of the simulated BRF and measured multi-angle reflectance; $\mathrm{SP}$ - solar principal plane; VP-the vertical solar principal plane.

winter wheat remained in the stage of seedling emergence and its roots had not reached $50 \mathrm{~cm}$ in depth, the soil volumetric water content could only be measured at a depth of $20 \mathrm{~cm}$ using the adopted TDR measurement. To test the correlation between the model estimated $\xi$ and the TDR measurements, a regression analysis was conducted for each of the 8 sets of $\xi$ values and the corresponding TDR measurements for each band, respectively. Based on this, the three most closely correlated bands were selected to establish a multi-angle and multi-band conversion (Fig. 5).

To compare the differences of estimation accuracy $\xi$ among a combination of three bands and a single band, the estimation results of the single band (Table 2) and the combined estimation results of R, NIR and SWIR2 were statistically analyzed with TDR measurements. The statistical correlation of the single band followed the order of NIR $>$ R $>$ SWIR $2>$ G $>$ SWIR 1 . The combined estimation accuracy of the three bands of R, NIR and SWIR2 was the highest $\left(R^{2}=0.867\right.$ and $\left.\mathrm{RMSE}=0.813\right)$.

\section{Conclusion}

This study investigated the directional radiation properties of farmland soil and extended the SOILSPECT directional model of soil radiation. The soil equivalent water thickness was introduced into the model by adopting a quantitative relationship between the dual-hemispherical reflectance and the single scattering albedo. Based on this, the SWAP-Hapke Soil Directional Radiation Model was established with consideration of the water content parameters.

A surface spectrometer obtained 8 sets of multi-angle spectral data, while the corresponding soil moisture data were measured. The model sensitivity analysis in Mode 1 showed that a relatively good stability existed between $\omega$ and $B_{0}$ with variation ranges of approximately 0.40 for both parameters; $h$ displayed relatively great variation. A sensitivity relationship of $b>b^{\prime}>c^{\prime}>c$ existed among the four parameters, $b, c, b^{\prime}$ and $c^{\prime}$, that influenced the soil properties of forward and backward scattering.

Model parameter estimations were carried out in the five characteristic bands by combining the Ant Colony Algorithm and Powell's algorithm, and the comparison was conducted among the estimation accuracies of model parameters with a single wavelength in Mode 2. Based on the accuracy statistics of RMSE and E, the overall estimation accuracy of model parameters was NIR $>$ SWIR $2>$ R $>$ SWIR $1>$ G. Their sequence was primarily influenced by the comprehensive effects of the atmospheric absorption and scattering characteristics of different bands, the sensitivity of soil moisture absorption and the spectrometer noises. The obtained soil moisture-related parameters solved with 8 sets of data varied due to the variability of soil moisture in the spatial distribution. Among them, 8 sets of $\omega$ estimates showed a relatively minor variation. A relationship of $b>b^{\prime}>\left|c^{\prime}\right| \approx|c|$ 


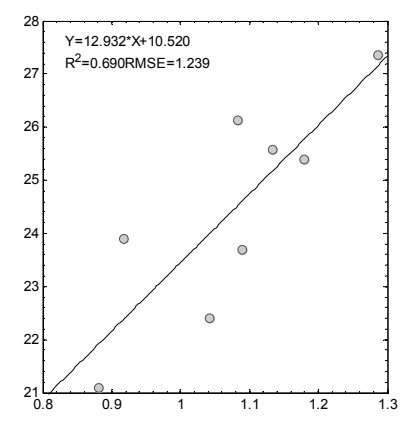

(a) G band

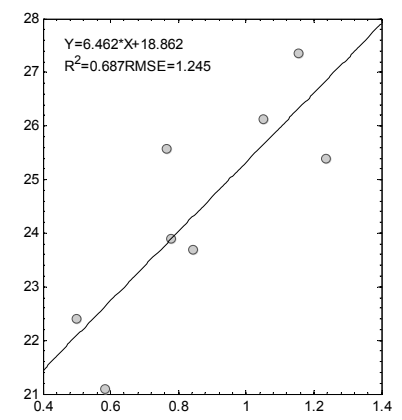

(e) SWIR1 band

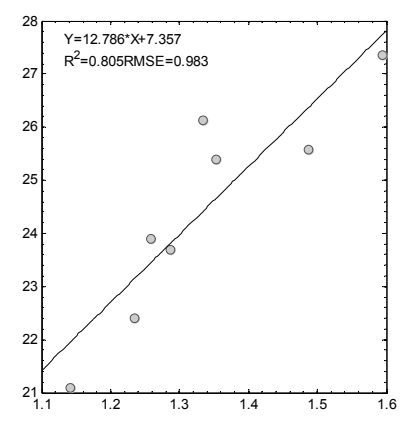

(b) R band

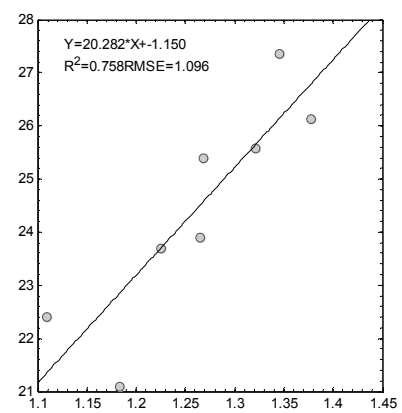

(f) SWIR2 band

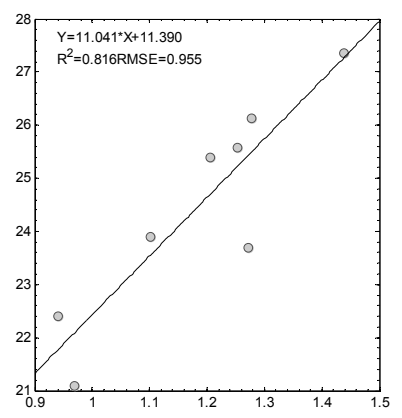

(c) NIR band

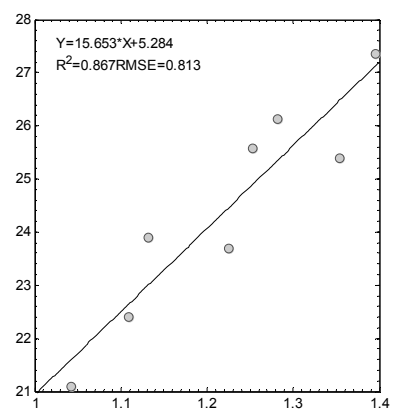

(g) R-NIR-SWIR2 bands

Fig. 5. The correlation analysis between the SWAP-Hapke-modeled $\xi$ and in situ TDR-measured soil volumetric water content (VWC). $\xi$ is the thickness of equivalent saturated liquid water. $\mathrm{X}$-axis is the estimated the thickness of equivalent saturated liquid water, and Y-axis is the in situ TDR measured VWC.

was present among the four parameters of $b, c, b^{\prime}$ and $c^{\prime}$, with the greatest variation range for $b$. Therefore, the soil exhibited a backward scattering property.

Adopting the SWAP-Hapke model parameters obtained through the combined solution of the three bands of $\mathrm{R}$ NIR-SWIR2 simulated a set of multi-angle spectral data, including the vertical solar principal plane and solar principal plane, which were compared and analyzed by combining the observation data. The model simulation values were consistent with the measured values. With a regression analysis of model estimated soil equivalent water thickness $\xi$ values and the corresponding TDR measurements, the correlation statistics showed NIR $>$ R $>$ SWIR2 $>$ G $>$ SWIR1, among which the correlation coefficients of NIR, R and SWIR2 were greater than 0.8. Based on the above conditions, the multi-angle spectral observation data from the three optimal bands were used for the solution of the same set of parameters, which were then statistically analyzed with the TDR data. The regression $\left(R^{2}=0.867\right.$, RMSE $\left.=0.813\right)$ was significantly better than the single statistical accuracies of the five bands, indicating that the increase of effective band numbers also contributed to the improvement of the estimation accuracy of soil water parameters to a certain extent under the conditions of multi-angle observation.
Table 3. The parameters of SWAP-Hapke model.

\begin{tabular}{lr}
\hline Variable(unit) & Designation \\
\hline Incident solar zenith angle(deg) & $\theta_{\mathrm{S}}$ \\
Sensor observation zenith angle(deg) & $\theta_{\mathrm{O}}$ \\
Relative azimuth between the sun and & $\phi$ \\
the observation direction(deg) & \\
Single scattering albedo of soil & $\omega$ \\
Scattering phase function & $P(g)$ \\
Coefficients of the scattering phase & $b, c, b^{\prime}, c^{\prime}$ \\
function & $B(g)$ \\
Backward scattering function & $B_{0}$ \\
Hot spot effect factor & $h$ \\
Soil roughness (cm) & $\xi$ \\
Equivalent water thickness(cm) & $\alpha$ \\
Absorption coefficient of water(cm & \\
The double-hemisphere reflectance of & $r_{\mathrm{W}}$ \\
wet soil & \\
The sampling dry soil reflectance & $r_{0}$ \\
\hline
\end{tabular}


Because the multi-angle measurement is a timeconsuming and energy-costing task, it is difficult to obtain a great amount of data in a short time. In this study, only 8 sets of multi-angle spectral data were measured in the two sampling areas without considering soil moisture gradient, soil types and other factors. Because of the uncertainties of the multi-angle spectral observation data, TDR measurements data, etc., the current conclusions are probably not universal and need further verification. Special efforts should undertake converting the quantitative relationship between the soil equivalent water thickness and soil water content obtained in the surface "spots" into that in the pixel "planes". Only in this way will the combined inversion of soil water content be conducted with surface observations and multi-angle and multi-spectral satellite data.

Acknowledgements. This study was supported by the Natural Science Foundation of China (40901173, 41071228), the Chinese State Key Basic Research Project (2011CB311806), the Beijing Municipal Natural Science Foundation (4102021), and the Open Funds of State Key Laboratory of Remote Sensing Science, jointly sponsored by the Institute of Remote Sensing Applications of Chinese Academy of Sciences and Beijing Normal University (OFSLRSS201109). We are grateful to the anonymous reviewers for their valuable comments and recommendations.

Edited by: C. de Michele

\section{References}

Bach, H. and Mauser, W.: Modeling and model verification of the spectral reflectance of soils under varying moisture conditions, Proceedings of IEEE IGARSS'94, 4, 2354-2356, 1994.

Baghdadi, N., Boyer, N., Todoroff, P., El Hajj, M., and Bégué, A.: Potential of SAR sensors TerraSAR-X, ASAR/ENVISAT and PALSAR/ALOS for monitoring sugarcane crops on Reunion Island, Remote Sens. Environ., 113(8), 1724-1738, 2009.

Chappell, A., Zobeck, T., and Brunner, G.: Using bi-directional soil spectral reflectance to model soil surface changes induced by rainfall and wind-tunnel abrasion, Remote Sens. Environ., 102, 328-343, 2006.

Cierniewski, J.: A model for soil surface roughness influence on the spectral response of bare soils in the visible and near infrared range, Remote Sens. Environ., 23, 98-115, 1987.

Cooper, K. D. and Smith, J. A.: A Monte Carlo Reflectance Model for Soil Surfaces with Three-Dimensional Structure, IEEE T. Geosci. Remote, GE-23(5), 668-673, 1985.

Dorigo, M., Birattari, M., and Stutzle, T.: Ant colony optimization artificial ants as a computational intelligence technique, IEEE Comput. Intell. M., 1(4), 28-39, 2006.
Dorigo, M., Maniezzo, V., and Colorni, A. Ant system: optimization by a colony of cooperating agents, IEEE Transaction on Systems, Man, and Cybernetics Part B, 26(1), 29-41, 1996.

Fabiano, P. and David, H. B.: Quantification of absorber through a scattering medium of different thickness using evanescent light piping, J. Near Infrared Spec., 18(1), 7-26, 2010.

Fuwei, P., Hideyuki S., Jiahua, W., Donghai, H., and Yukihiro, O.: Partial least square regression with variable-bagged model ensemble, J. Near Infrared Spec., 17(1), 33-40, 2009.

Hapke, B. W.: Bidirectional reflectance spectroscopy: 1. Theory, J. Geophys. Res., 86, 3039-3054, 1981.

Jacquemoud, S., Bater, F., and Hanocq, J. F.: Modeling spectral and bidirectional soil reflectance, Remote Sens. Environ., 41, 123132, 1992.

Joseph, A., Velde van der, R., O’Neill, P. E., Lang, R. H., and Gish, T.: Soil moisture retrieval during a corn growth cycle using Lband (1.6 GHz) Radar observations, IEEE T. Geosci. Remote, 46(8), 2365-2374, 2009.

Li, X. W. and Strahler, A. H.: Geometric-optical bidirectional reflectance modeling of mutual shadowing effects of crown in a forest canopy, IEEE T. Geosci. Remote, 30(2), 276-292, 1992.

Li, X. W. and Wang, J. D.: Model of vegetation Optical remote sensing and vegetation structural parameter, Science Press, Beijing, 1995.

Liang, S. L.: Quantitative Remote Sensing of Land Surfaces. New Jersey: John Wiley, 2004.

Liu, W. D., Fredéric, B., Zhang, B., Zheng, L. F., and Tong, Q. X.: Extraction of soil moisture information by hyperspectral remote sensing, Acta Pedologica Sinica, 41(5), 700-706, 2004.

Matlab. Users guide: Optimisation toolbox. Version 2.1 (Release 12), Mathworks Inc., 2000.

Norman, J. M., Welles, J. M., and Walter, E. A.: Contrast among bidirectional reflectance of leaves, canopies, and soils, IEEE T. Geosci. Remote, 23, 659-667, 1985.

Pinty, B. and Verstraete, M. M.: Extracting information on surface properties from bidirectional reflectance measurements, J. Geophys. Res., 96, 2865-2874, 1991.

Pinty, B., Verstraete, M. M., and Dickinson, R. E.: A physical model for predicting bidirectional reflectances over bare soil., Remote Sens. Environ., 27, 273-288, 1989.

Price, J.: On the information content of soil reflectance spectra, Remote Sens. Eviron., 33, 113-121, 1990.

Segelstein, D.: The Complex Refractive Index of Water, M.S. Thesis, University of Missouri-Kansas City, USA., 1981.

Verhoef, W. and Hapke, B.: Coupled soil-leaf-canopy and atmosphere radiative transfer modeling to simulate hyperspectral multi-angular surface reflectance and TOA radiance data, Remote Sens. Environ., 109(2), 166-182, 2007.

Walthall, C. L., Norman, J. M., Blad, B. L., Welles, J. M., and Campbell, G.: Simple equation to approximate the bidirectional reflectance from vegetative canopies and bare soil surfaces, Appl. Opt., 24, 383-387, 1985.

Wu, Y. Z., Peng, G., Qiang, L., and Chappell, A.: Retrieving photometric properties of desert surfaces in China using the Hapke model and MISR data, Remote Sens. Environ., 113(1), 213-223, 2009. 